\title{
Thermometry and cooling of a Bose gas to 0.02 times the condensation temperature
}

\author{
Ryan Olf ${ }^{1 \star}$, Fang Fang ${ }^{1}$, G. Edward Marti ${ }^{1 \dagger}$, Andrew MacRae' and Dan M. Stamper-Kurn ${ }^{1,2}$
}

\begin{abstract}
Trapped quantum gases can be cooled to impressively low temperatures ${ }^{1,2}$, but it is unclear whether their entropy is low enough to realize phenomena such as $d$-wave superconductivity and magnetic ordering ${ }^{3}$. Estimated critical entropies per particle for quantum magnetic ordering are $\sim 0.3 k_{\mathrm{B}}$ and $\sim 0.03 k_{B}$ for bosons in three- and two-dimensional lattices, respectively ${ }^{4}$, with similar values for Néel ordering of latticetrapped Fermi gases ${ }^{5}$. Here we report reliable single-shot temperature measurements of a degenerate $\mathbf{R b}$ gas by imaging the momentum distribution of thermalized magnons, which are spin excitations of the atomic gas. We record average temperatures fifty times lower than the Bose-Einstein condensation temperature, indicating an entropy per particle of $\sim 0.001 k_{\mathrm{B}}$ at equilibrium, nearly two orders of magnitude lower than the previous best in a dilute atomic gas ${ }^{2,6}$ and well below the critical entropy for antiferromagnetic ordering of a Bose-Hubbard system. The magnons can reduce the temperature of the system by absorbing energy during thermalization and by enhancing evaporative cooling, allowing the production of low-entropy gases in deep traps.
\end{abstract}

In many experiments on strongly interacting atomic-gas systems, the low-entropy regime is reached by first preparing a weakly interacting bulk Bose gas at the lowest possible temperature, and then slowly transforming the system to become strongly interacting $^{7-11}$. To discern whether the transformation is adiabatic and to determine indirectly the thermodynamic properties of the strongly interacting system, the system is returned to the weakly interacting regime where relations between temperature, entropy and other properties are known. Therefore, methods to lower entropies and measure temperatures of weakly interacting gases are important for the study of both weakly and strongly interacting atomic-gas systems.

In this Letter, we report cooling a Bose gas to a few per cent of the condensation temperature, $T_{c}$, corresponding to an entropy per particle $S / N \approx 1 \times 10^{-3} k_{\mathrm{B}}$, where $k_{\mathrm{B}}$ is the Boltzmann constant. Surprisingly, we achieve this low entropy using a standard technique: forced evaporation in an optical dipole trap, which we find remains effective in a previously uncharacterized regime. The lowest temperatures we report are achieved at very shallow final trap depths, as low as $20 \mathrm{nK}$, set by stabilizing the optical intensity with a longterm fractional reproducibility better than $10^{-2}$. In addition, we demonstrate and characterize a method of cooling that lowers the entropy without changing the trap depth, possibly allowing the lowentropy regime to be reached or maintained in systems where the trap depth is constrained.

Both thermometry and cooling require a means of distinguishing thermal excitations. For example, forced evaporative cooling ${ }^{12,13}$ depends on the ability to selectively expel high-energy excitations from the system. Similarly, thermometry of a degenerate quantum gas requires one to identify the excitations that distinguish a zero-temperature from a non-zero-temperature gas. Both these tasks become difficult when $S / N$, or, equivalently, the fraction of thermal excitations $N_{\text {th }} / N$, is small ${ }^{14}$. Time-of-flight temperature measurements, in which the gas is released from the trap and allowed to expand before being imaged, have required $N_{\text {th }} / N$ of at least several percent, limiting such thermometry of Bose gases to $T \geq 0.3 T_{c}$ and of Fermi gases to $T \geq 0.05 T_{F}$, where $T_{F}$ is the Fermi temperature.

We extend thermometry to the deeply degenerate regime of a Bose gas by measuring the momentum distribution of a small number of spin excitations, similar to the co-trapped impurity thermometry commonly employed in Fermi gases ${ }^{15-18}$. Even in a highly degenerate Bose gas with a vanishingly small noncondensed fraction, the minority spin population can be made dilute enough to remain non-degenerate and thereby carry a large entropy and energy per particle. Furthermore, the minority spins that we use-magnons within a ferromagnetic spinor Bose-Einstein condensate-support a higher number of thermal excitations than the majority spins because of their free-particle density of states ${ }^{19}$, increasing the signal of the temperature measurement. Performing spin-selective measurements on the minority spins allows the gas temperature to be readily determined.

Our procedure to measure temperatures is illustrated in Fig. 1a. Experiments begin with spin-polarized ${ }^{87} \mathrm{Rb}$ in the $\left|F=1, m_{F}=-1\right\rangle$ state confined in an anisotropic optical dipole trap and cooled to just above quantum degeneracy by forced evaporation to an intermediate trap depth. The spin quantization axis is defined by a $180 \mathrm{mG}$ magnetic field. We tip the gas magnetization with a brief radiofrequency $(\mathrm{RF})$ pulse, coherently transferring a small fraction (up to $15 \%$ ) of the atoms primarily into the $\left|F=1, m_{F}=0\right\rangle$ state, creating magnons that rapidly decohere and thermalize. The gas is then cooled further by forced evaporation to a final trap depth where the temperature reaches a steady state.

Next, we release the gas from the optical trap and image the momentum distribution of the magnons. On extinguishing the trap light, the gas expands rapidly in the most tightly confined (vertical) direction, quickly reducing the outward pressure within the gas. We then use a sequence of microwave and optical pulses to drive away atoms not in the $\left|m_{F}=0\right\rangle$ Zeeman state. Finally, we transfer the remaining atoms to an atomic state $\left(\left|F=2, m_{F}=1\right\rangle\right)$ suitable for two-dimensional magnetic focusing ${ }^{20}$ so that their transverse spatial distribution, which we image, closely reflects their initial transverse velocity distribution. The majority gas is probed by imaging $\left|m_{F}=0\right\rangle$ atoms immediately after the application of an RF pulse.

${ }^{1}$ Department of Physics, University of California, Berkeley, California 94720, USA. ${ }^{2}$ Materials Sciences Division, Lawrence Berkeley National Laboratory, Berkeley, California 94720, USA. PPresent address: JILA, National Institute of Standards and Technology and University of Colorado, Boulder,

Colorado 80309-0440, USA. *e-mail: ryan@efrus.com 


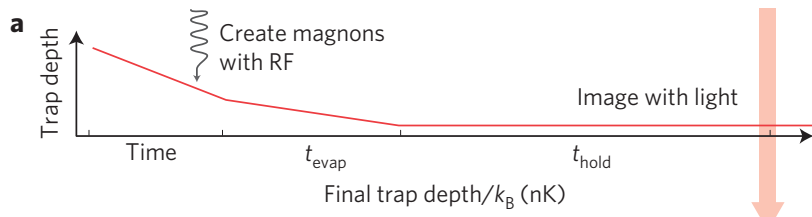

b

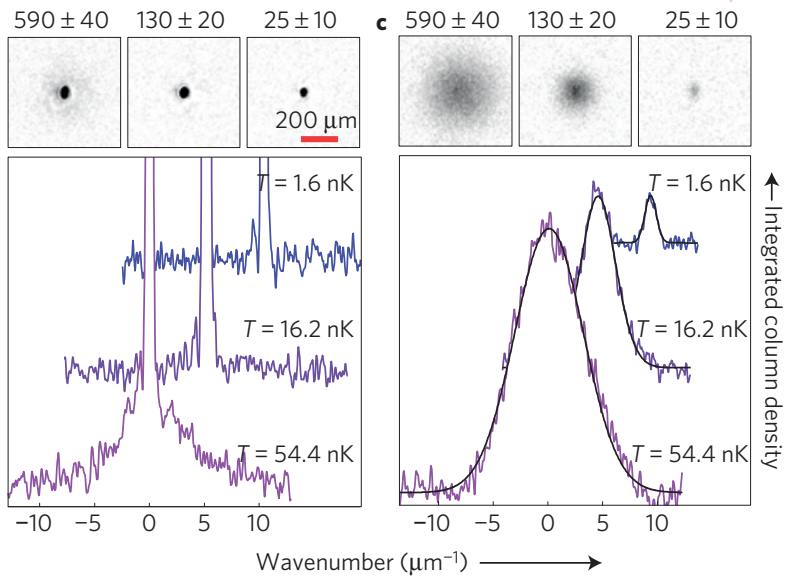

Figure 1 | Magnon thermometry. a, Magnons are created and decohere rapidly in a non-degenerate spinor Bose gas at an intermediate trap depth. Forced evaporative cooling to a final, variable, trap depth reduces the temperature and the majority gas undergoes Bose-Einstein condensation. b,c, Images and corresponding integrated line profiles of the momentum distribution of the majority (b) and magnon gas (c) are each shown at three different final trap depths. Line profiles are shown offset for clarity. A condensate obscures the momentum distribution of the non-condensed fraction of the majority gas, especially at low temperatures. In contrast, the magnons can have little to no condensed fraction, allowing non-condensed magnons to be identified and the temperature determined.

The advantage of using incoherent spin excitations to measure temperature is exhibited in Fig. 1, which compares the momentum distribution of the majority gas (b) to that of the magnons (c). At $T \lesssim 0.3 T_{c}$, the non-condensed fraction of the majority gas is obscured by the condensed fraction, the distribution of which is broadened owing to interactions and imaging resolution. In contrast, the temperature can be easily extracted from the momentum distribution of the thermalized magnons. Temperatures extracted from the magnons (Fig. 2a) agree with those extracted from the majority gas under conditions where both momentum distributions can be measured. At lower temperatures, the magnon thermometer allows measurements in a hitherto unmeasurable regime.

Our measurements reveal that forced evaporative cooling produces Bose gases with extremely low temperature and entropy. At the lowest trap-depth setting (dashed circles, Fig. 2), we measure an average gas temperature of $T=1.04(3)_{\text {stat }}(7)_{\text {sys }} \mathrm{nK}$, corresponding to $T / T_{\mathrm{c}}=0.022(1)(2)$, where $T_{\mathrm{c}}$ is calculated using the measured atom number, $N=8.1 \times 10^{5}$, and optical trap frequencies. The numbers in parentheses indicate statistical and systematic uncertainties in the last decimal place, respectively. For this gas, $k_{\mathrm{B}} T / \mu \approx 0.07$, with $\mu$ the chemical potential, meaning that thermal excitations in the high-density region of the condensed gas (in the majority spin state) are predominantly phonons. Measurement procedures, error estimates and calculations of $T_{\mathrm{c}}$ and $\mu$ are detailed in Methods.

We observe that evaporative cooling is highly efficient with respect to particle loss in the regime $\mu / k_{\mathrm{B}}<T<T_{\mathrm{c}}$ (Fig. 2b). When $T<\mu / k_{\mathrm{B}}$, the cooling efficiency reduces, consistent with the fact that the chemical potential accounts for a non-negligible amount of the energy carried away by each atom lost to evaporation. The relevance of the chemical potential in evaporation is also indicated in Fig. $2 \mathrm{a}$ by the observation that $\eta \equiv U /\left(k_{\mathrm{B}} T\right)$, the ratio of trap depth to thermal energy, increases at lower trap depths, and that the temperature at a fixed trap depth depends on the number of atoms. In an evaporatively cooled gas, the temperature responds to the effective trap depth, $U_{\text {eff }}=U-\mu$, the potential energy depth minus the chemical potential, rather than the trap depth alone, and in the regime $T<\mu / k_{\mathrm{B}}$, the difference $U-U_{\text {eff }}=\mu$ is significant.

We calculate the Bogoliubov energy spectrum of the confined quantum degenerate gas, including the effects of trap anharmonicity, and find its entropy per particle at equilibrium (see Methods) to be $S / N=1 \times 10^{-3} k_{\mathrm{B}}$, the lowest value ever reported for an atomic gas. By comparison, the $500 \mathrm{pK}$ Bose gas reported in ref. 1 has $S / N=3.6 k_{\mathrm{B}}\left(T / T_{\mathrm{c}}\right)^{3} \sim 1.5 k_{\mathrm{B}}$ using relations for a non-interacting gas at $T / T_{\mathrm{c}} \sim 0.75$. Other reported values include $S / N \sim 0.05 k_{\mathrm{B}}$ at the centre of a resonantly interacting Fermi gas $^{6}, S / N=0.27 k_{\mathrm{B}}$ (ref. 21 ) or $\sim 0.1 k_{\mathrm{B}}$ (ref. 2) for bosons in a lattice, and $T / T_{\mathrm{c}} \sim 0.15$ in a double-well Bose-Einstein condensate ${ }^{22}$. Thermometry based on imaging incoherent phonons indicated a temperature around seven times higher than reported here in a ${ }^{87} \mathrm{Rb}$ condensate of similar density ${ }^{23}$.

Having magnons present during evaporative cooling reduces the temperature of the trapped gas by increasing the efficacy of evaporative cooling. Forced evaporative cooling from a trap with an effective trap depth $U_{\text {eff }} \gg k_{\mathrm{B}} T$ has a cooling power proportional to the number of thermal excitations with excitation energies above $U_{\text {eff }}$. In a weakly interacting single-component degenerate Bose gas, the number of thermal excitations is determined by the temperature and is independent of the total particle number, fixing the evaporative cooling rate. By seeding the gas with additional spin excitations at constant total particle number, the total number of thermal excitations, and thus the evaporative cooling power, increases.

We observe that, for $T \gtrsim \mu / k_{\mathrm{B}}$, this magnon-assisted evaporation leads to $T / T_{\mathrm{c}}$ lower than that reached by single-component evaporation at the same final particle number (' $x$ ' points in Fig. $2 b$ ). When $T \lesssim \mu / k_{\mathrm{B}}$, we find magnons not to reduce the temperature, perhaps because of the small number of non-condensed magnons and the decrease in evaporative cooling efficiency in general. Magnon-assisted evaporation is also evidenced by the fact that the per-atom loss rate is higher for the magnon gas than for the majority gas: for both populations, the atom loss reflects the selective evaporative loss of thermal excitations. For the majority atoms, thermal excitations are just a small fraction of the total population, whereas they represent a much higher fraction of the magnons.

Unbiased thermometry of the majority gas is performed by extrapolating measurements with various numbers of magnons to the zero-magnon limit (Fig. 2a inset). In addition, by examining evaporative cooling in a state-dependent optical trap, we have confirmed that the temperature indicated by the magnons varies with the trap depth of the majority gas, even for a fixed trap depth of atoms in the $\left|m_{F}=0\right\rangle$ state. This observation gives further confidence that the magnon thermometer accurately records the temperature of the majority gas.

The same characteristics that make dilute magnons a good thermometer-high relative energy and entropy per particleenable them to cool without lowering the trap depth, beyond what can be achieved by magnon-assisted evaporation. Spin excitations created in a degenerate Bose gas decrease its temperature by a process known as decoherence cooling ${ }^{24}$ or demagnetization cooling ${ }^{2,25}$. Immediately after the RF pulse is applied, the magnon population has the same energy and momentum distribution as the initially polarized degenerate Bose gas, with a large condensed fraction, which carries no energy above the chemical potential, and a small normal fraction, which carries on the order of $k_{\mathrm{B}} T$ of energy per particle. On thermalization, the normal fraction within the magnon gas increases, bounded from above by its critical number for condensation, and the energy and entropy of the magnons increases. The energy gained by the magnons is energy lost by the 

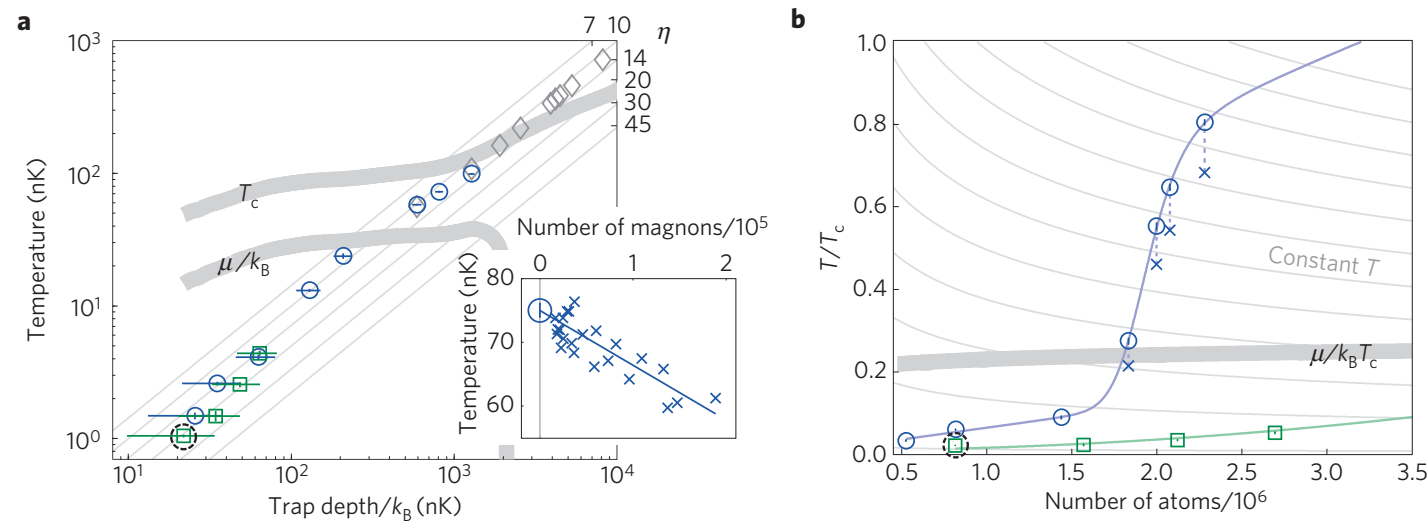

Figure 2 | Thermometry results. a,b, Two runs (blue circles, green squares) differ in initial atom number (about three and five million atoms at $T=T_{\mathrm{C}}$ respectively). $T(\mathbf{a})$ and $T / T_{\mathrm{c}}(\mathbf{b})$ are measured at various optical trap depths. Lower $T$ and $T / T_{\mathrm{c}}$ are achieved in runs with larger initial atom number. a, Thermometry using the majority gas (light grey diamonds) agree with the thermalized magnon thermometry extrapolated to the zero-magnon values (circles and squares). Error bars show the statistical uncertainty of extrapolated temperature (vertical) and the systematic uncertainty in trap depth (horizontal). Thin diagonal grey lines show contours of $\eta$, the ratio of trapping potential depth to temperature. Inset: the magnon-free temperature is determined by extrapolating single-shot temperatures ('crosses') versus total number of magnons imaged, here at trap depth of $k_{\mathrm{B}} \times 800 \mathrm{nK}$, to the zero-magnon limit (circle). A similar extrapolation determines the value of $T / T_{\mathrm{C}}$ achieved by evaporation only. $\mathbf{b}$, Efficiency of evaporation only (circles, squares), with error bars showing statistical uncertainty of the extrapolated $T / T_{\mathrm{C}}$, is compared against magnon-assisted evaporation ('crosses'). Solid lines connecting points are guides to the eye, with a steeper slope indicating more efficient evaporation. Dotted lines connect corresponding points at the same trap depth. The thick grey lines showing $\mu / k_{\mathrm{B}} T_{\mathrm{C}}$ are calculated using the frequencies of the $k_{\mathrm{B}} \times 60 \mathrm{nK}$ deep trap.

a
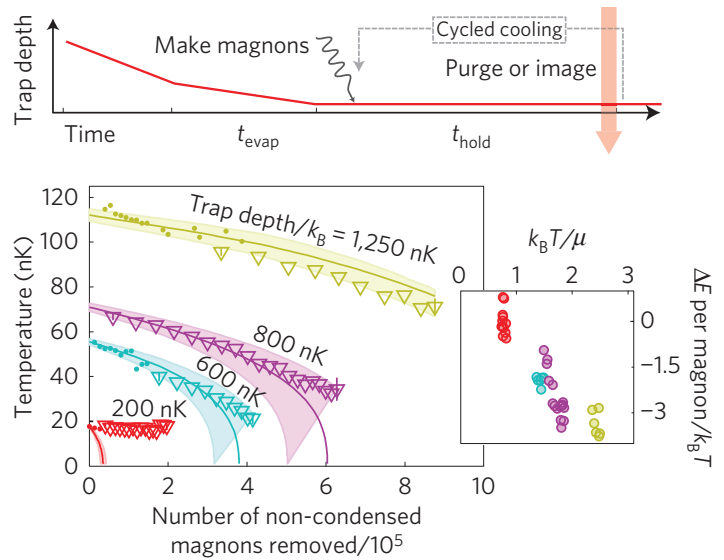

c

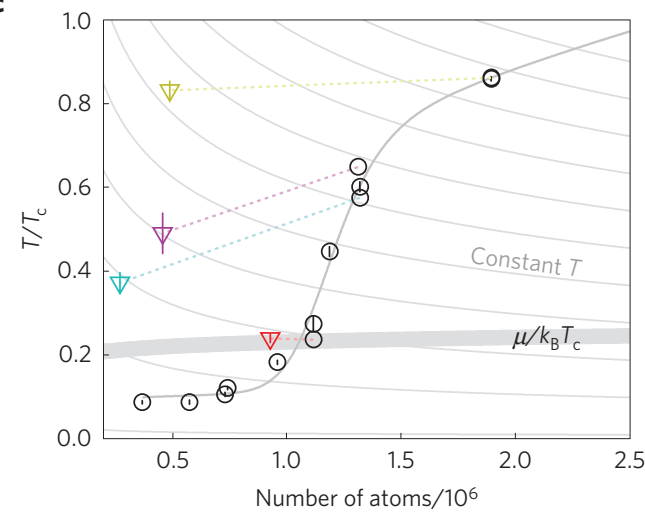

Figure 3 | Cycled decoherence cooling. a, Magnons are created at the final trap depth and cool the gas as they thermalize. Thermalized magnons can be removed from the trap and the cooling process repeated before measuring the temperature by imaging the momentum distribution of the thermalized magnons. b. Each non-condensed magnon removed from the trap takes energy from the gas. Decoherence cooling trajectories are plotted versus cumulative number of magnons removed. Filled circles show temperatures after a single cycle of decoherence, but with varying numbers of magnons. Some additional magnon-assisted evaporative cooling may also be present. Open triangles show repeated cycles of magnon creation, thermalization and purge, with representative error bars on the first and last triangles giving statistical error over several repetitions. Solid lines show zero-free-parameter theory predictions assuming each non-condensed magnon removes $3 \mathrm{k}_{\mathrm{B}} T$ energy. Shaded areas indicate the range of predictions included within the uncertainty in the cumulative number of magnons removed (we image $75 \% \pm 10 \%$ of the magnons present). Inset: the amount of energy removed by each magnon, in units of $k_{B} T$, is plotted versus the ratio of $\mu / k_{B} T$, where each point is based on an estimate of the slope over four consecutive triangle points. We observe that the net energy carried away by magnons vanishes when $T \lesssim \mu / k_{\mathrm{B}}$. c. Efficiency of evaporation only (open circles) is compared against cycled decoherence cooling (open triangles), with error bars representing statistical uncertainty. The solid line between circles is a guide to the eye. Dotted lines connect corresponding points at the same trap depth. Compared to evaporation, decoherence cooling can reach considerably lower $T / T_{\mathrm{C}}$ at the same trap depth in the regime $T \gtrsim \mu / k_{\mathrm{B}} T$ and $T / T_{\mathrm{c}} \lesssim 0.9$.

majority gas, as the entire process, apart from the slight effects of magnetic field inhomogeneities and dipolar interactions, occurs at constant energy. In Fermi gas systems, a similar process would lead to heating as the energy and entropy per particle of the minority spins is less than that of the majority gas at equilibrium.

Whereas the overall entropy rises during thermalization, the entropy per particle of the majority gas may decrease. Considering a non-interacting harmonically trapped gas, we calculate that decoherence cooling would reduce $T / T_{\mathrm{c}}$ of the majority gas only when $T / T_{c}<\sqrt[3]{(3 \zeta(3)) /(4 \zeta(4))}=0.94$, where $\zeta$ is the Riemann zeta function, consistent with our observations.

To demonstrate the capabilities of decoherence cooling, we introduce a variable number of magnons at the final trap depth and allow them to evolve towards thermal equilibrium with the assistance of a weak magnetic field gradient of $0.2-2 \mathrm{mG} \mathrm{cm}^{-1}$ (for more on magnon thermalization, see Supplementary Discussion). Then, 
as illustrated in Fig. 3a, we either measure the temperature or expel the magnons using a spin-selective process, leaving a colder spinpolarized condensed gas. The decoherence cooling process can then be repeated to further cool the sample, with the number of magnons, magnetic field gradient and thermalization time optimized at each repetition. A single pass of decoherence cooling, using pseudo-spins in a degenerate ${ }^{87} \mathrm{Rb}$ gas, has previously been shown to slightly reduce the temperature, but not $T / T_{c}$, of the resulting mixture ${ }^{24}$.

Whereas the thermal energy $k_{\mathrm{B}} T$ achieved by forced evaporative cooling of a single-component gas is limited to around $1 / \eta \sim 1 / 10$ of the trapping potential depth, the temperatures reached by cycled decoherence cooling are far lower. For example, we produced a $20 \mathrm{nK}$ gas within a $k_{\mathrm{B}} \times 600 \mathrm{nK}$ deep potential, corresponding to $1 / \eta=1 / 30$, and reduced $T / T_{\mathrm{c}}$ substantially, from 0.58 to 0.37 (cyan triangles, Fig. $3 b, c)$. On the other hand, decoherence cooling is generally less efficient in terms of atom loss. An atom lost through evaporative cooling removes an energy $\sim \eta k_{\mathrm{B}} T$, whereas a magnon brought to equilibrium and forcibly ejected removes only $3 k_{\mathrm{B}} T$ for a non-interacting gas, consistent with our observations when $k_{\mathrm{B}} T \gg \mu$. At lower temperatures, we expect each magnon to remove less energy, 1 to $1.5 k_{\mathrm{B}} T$, as interactions and quantization of the trap modes become more important. Even so, cooling would be expected, in the ideal case, to become increasingly effective as the heat capacity drops. In contrast, we find decoherence cooling to be ineffective at reducing both $T$ and $T / T_{\mathrm{c}}$ in the low-temperature regime $k_{\mathrm{B}} T \lesssim \mu$ (Fig. 3b,c, inset and red triangles), perhaps owing to energy deposited in the trapped gas during the magnon expulsion and by heating processes, which become more significant as thermalization times increase.

In principle, the process of creating the spin mixture employed in decoherence cooling can heat the gas by changing its interaction energy. However, neglecting small magnetic dipolar interactions, the magnon is a gapless Goldstone excitation corresponding to the rotational symmetry of real spins ${ }^{19}$. Accordingly, the $s$-wave scattering lengths that characterize the interactions between two majority-spin atoms, $a_{-1,-1}$, and between a majority and minorityspin atom, $a_{-1,0}$, are identical. In contrast, spin excitations involving a different hyperfine state can deposit energy into the condensate owing to a mismatch of $s$-wave scattering lengths.

In this work, we have demonstrated the use of spin excitations within a highly degenerate Bose gas to measure and reduce the temperature. With our improved thermometry, we observe a record low entropy of $1 \times 10^{-3} k_{\mathrm{B}}$ per particle for a spinpolarized Bose-Einstein condensate, illustrating the power of standard cooling techniques to reach the long-sought low-entropy regime. Magnon-assisted evaporation and cycled decoherence cooling should work for other spin or pseudospin mixtures of Bose gases so long as the gas thermalizes at constant minority particle number, providing a flexible platform for creating or maintaining low entropies in deep traps.

\section{Methods}

Methods and any associated references are available in the online version of the paper.

Received 24 February 2015; accepted 17 June 2015; published online 20 July 2015; corrected online 20 August 2015

\section{References}

1. Leanhardt, A. E. et al. Cooling Bose-Einstein condensates below 500 picokelvin. Science 301, 1513 (2003).

2. Medley, P., Weld, D. M., Miyake, H., Pritchard, D. E. \& Ketterle, W. Spin gradient demagnetization cooling of ultracold atoms. Phys. Rev. Lett. 106, 195301 (2011).
3. Bloch, I., Dalibard, J. \& Nascimbene, S. Quantum simulations with ultracold quantum gases. Nature Phys. 8, 267 (2012).

4. Capogrosso-Sansone, B., Söyler, S. G., Prokof'ev, N. V. \& Svistunov, B. V. Critical entropies for magnetic ordering in bosonic mixtures on a lattice. Phys. Rev. A 81, 053622 (2010).

5. Paiva, T., Loh, Y. L., Randeria, M., Scalettar, R. T. \& Trivedi, N. Fermions in 3D optical lattices: Cooling protocol to obtain antiferromagnetism. Phys. Rev. Lett. 107, 086401 (2011).

6. Ku, M. J. H., Sommer, A. T., Cheuk, L. W. \& Zwierlein, M. W. Revealing the superfluid lambda transition in the universal thermodynamics of a unitary Fermi Gas. Science 335, 563 (2012).

7. Greiner, M., Mandel, O., Esslinger, T., Hänsch, T. W. \& Bloch, I. Quantum phase transition from a superfluid to a Mott insulator in a gas of ultracold atoms. Nature 415, 39-44 (2002).

8. Bakr, W. S. et al. Probing the superfluid-to-Mott insulator transition at the single-atom level. Science 329, 547-550 (2010).

9. Paredes, B. et al. Tonks-Girardeau gas of ultracold atoms in an optical lattice. Nature 429, 277-281 (2004).

10. Trotzky, S. et al. Time-resolved observation and control of superexchange interactions with ultracold atoms in optical lattices. Science 319, 295-299 (2008)

11. Spielman, I., Phillips, W. \& Porto, J. Mott-Insulator transition in a two-dimensional atomic Bose gas. Phys. Rev. Lett. 98, 080404 (2007).

12. Luiten, O. J., Reynolds, M. W. \& Walraven, J. T. M. Kinetic Theory of evaporative cooling of a trapped gas. Phys. Rev. A 53, 381 (1996).

13. Ketterle, W. \& van Druten, N. Evaporative Cooling of Trapped Atoms Vol. 37, 181-236 (Academic Press, 1996).

14. McKay, D. C. \& DeMarco, B. Cooling in strongly correlated optical lattices: Prospects and challenges. Rep. Prog. Phys. 74, 054401 (2011).

15. Regal, C. \& Jin, D. Experimental realization of the BCS-BEC crossover with a Fermi gas of atoms. Adv. At. Mol. Opt. Phys. 54, 1-79 (2007).

16. Nascimbène, S., Navon, N., Jiang, K. J., Chevy, F. \& Salomon, C. Exploring the thermodynamics of a universal Fermi gas. Nature 463, 1057-1060 (2010).

17. Spiegelhalder, F. M. et al. Collisional stability of ${ }^{40} \mathrm{~K}$ immersed in a strongly interacting Fermi gas of ${ }^{6}$ Li. Phys. Rev. Lett. 103, 223203 (2009).

18. McKay, D. \& DeMarco, B. Thermometry with spin-dependent lattices. New J. Phys. 12, 055013 (2010).

19. Marti, G. E. et al. Coherent magnon optics in a ferromagnetic spinor Bose-Einstein condensate. Phys. Rev. Lett. 113, 155302 (2014).

20. Tung, S., Lamporesi, G., Lobser, D., Xia, L. \& Cornell, E. Observation of the presuperfluid regime in a two-dimensional Bose gas. Phys. Rev. Lett. 105, 230408 (2010).

21. Bakr, W. S. et al. Orbital excitation blockade and algorithmic cooling in quantum gases. Nature 480, 500-503 (2011).

22. Gati, R. et al. A primary noise thermometer for ultracold Bose gases. New J. Phys. 8, 189-189 (2006).

23. Schley, R. et al. Planck distribution of phonons in a Bose-Einstein condensate. Phys. Rev. Lett. 111, 055301 (2013).

24. Lewandowski, H. J., McGuirk, J. M., Harber, D. M. \& Cornell, E. A. Decoherence-driven cooling of a degenerate spinor Bose gas. Phys. Rev. Lett. 91, 240404 (2003).

25. Fattori, M. et al. Demagnetization cooling of a gas. Nature Phys. 2 , 765-768 (2006).

\section{Acknowledgements}

We thank H. Kadau and E. Copenhaver for assistance improving the experimental apparatus. We acknowledge the primary research support from NASA and the AFOSR through the MURI program, and also secondary support for personnel through the NSF G.E.M. acknowledges support from the Fannie and John Hertz Foundation.

\section{Author contributions}

All authors provided experimental support and commented on the manuscript. Experimental data were acquired by R.O. and F.F., and analysed by R.O. G.E.M. conceived and performed preliminary experiments with cycled decoherence cooling. The manuscript was prepared by R.O. and D.M.S.-K. R.O. performed the calculations of entropy per particle. D.M.S.-K. supervised all work.

\section{Additional information}

Supplementary information is available in the online version of the paper. Reprints and permissions information is available online at www.nature.com/reprints.

Correspondence and requests for materials should be addressed to R.O.

\section{Competing financial interests}

The authors declare no competing financial interests. 


\section{Methods}

Optical trapping. Experiments were performed on ${ }^{87} \mathrm{Rb}$ gases in a single-beam optical dipole trap ${ }^{19}$. Evaporative cooling was realized by gradually lowering the depth of the optical trap. The optical trap was formed by light with a wavelength of $1,064 \mathrm{~nm}$, brought to a cylindrical focus at the location of the atoms. The optical trap was highly anisotropic, with trapping frequencies having a typical ratio 1:15:140, with the tightest confinement in the vertical direction. At low optical powers, the optical dipole trap is influenced by gravity so that the ratio of trap frequencies differs slightly. Trap frequencies were measured at many optical powers and are described well by a simple model of the trapping potential that accounts for the Gaussian focus and force of gravity. This model was used to estimate the trap depth. Systematic uncertainty in the trap depth is derived from the uncertainty in the parameters of the model.

The bulk of our experiments were performed with linearly polarized trap light, which imparts an equal dipole force on all Zeeman sublevels of $F=1{ }^{87} \mathrm{Rb}$. To verify that our temperatures vary with the trap depth of the majority gas, rather than that of the minority spins, we also performed experiments with circularly polarized trap light, which applies a differential force to atoms in different Zeeman sublevels. Using this circularly polarized trap, we vary the trap depth of the majority gas without affecting that of the $\left|m_{F}=0\right\rangle$ atoms by preparing the majority gas in either of the $\left|m_{F}=+1\right\rangle$ or $\left|m_{F}=-1\right\rangle$ states.

The lifetime and heating rate of the atoms in the optical trap are consistent with spontaneous scattering of the far-detuned trapping light.

State purification and preparation. About one millisecond after extinguishing the optical trapping light, we initiate the imaging sequence by purging atoms in the $\left|F=1, m_{\mathrm{F}}=-1\right\rangle$ state-the majority component of our gas in most of our experiments - using a series of alternating microwave pulses (resonant with the $\left|F=1, m_{F}=-1\right\rangle \rightarrow\left|F=2, m_{F}=0\right\rangle$ transition) and light pulses (resonant with the $F=2$ hyperfine manifold).

The number and strength of the optical/microwave pulses is optimized to expel all of the $\left|F=1, m_{F}=-1\right\rangle$ atoms with minimal impact on the number of atoms in the $\left|F=1, m_{F}=0\right\rangle$ state. Regardless, the purge process removes between $10 \%$ and $40 \%$ of the $\left|F=1, m_{F}=0\right\rangle$ atoms, depending on the number of $\left|F=1, m_{F}=-1\right\rangle$ atoms being purged and other experimental parameters, but it does not visibly impact the momentum of the atoms that remain. We estimate the number of magnons from the images by accounting for this loss, allowing considerable uncertainty.

Finally, we employ a calibrated microwave sweep to transfer atoms in the $\left|F=1, m_{F}=0\right\rangle$ state to the magnetically trappable $\left|F=2, m_{F}=1\right\rangle$ state with greater than $95 \%$ efficiency.

Magnetic focusing. The magnetic focusing lens takes the form of a magnetic potential with negligible curvature along the vertical (imaging) axis and weak, harmonic curvature in the image plane. The resulting magnetic trap causes atoms with momenta $\mathbf{p}$ in the image plane to converge on the real-space point $\mathbf{x}_{p}=\mathbf{p} / m \omega$ after a time $t_{\text {foc }}=2 \pi / 4 \omega$, where $m$ is the atomic mass and $\omega$ the trap frequency for atoms in the $\left|F=2, m_{F}=1\right\rangle$ state. The vertical gradient is selected to cancel the effect of gravity, and the trap frequency $\omega$ is chosen such that the atomic distribution is resolvable by our imaging system at the lowest accessible temperatures while still allowing us to image temperatures near $T_{c}$.

Uncertainty in the parameter $\omega$ is the largest source of systematic error in the temperatures reported in this work. Our measurements found $\omega=2 \pi \times 2.88 \pm 0.09 \mathrm{rad} \mathrm{s}^{-1}$ and the relation $\mathbf{x}_{p}=\mathbf{p} / m \omega$ was verified by comparing the amplitude and phase of centre-of-mass oscillations of our gas imaged both in situ and after application of the magnetic focusing lens.

Time-of-flight expansion of the gas in the unconfined vertical direction along with misalignment of the trap, imaging and focusing axes can lead to several types of aberration. For example, any projection of the vertical extent of the gas into the imaging plane will broaden the imaged column density. Such aberrations can lead to an overestimate of the temperature of the gas and are most pertinent at low temperatures. Trap, imaging and focusing axes are all aligned to gravity in our system. Good alignment is evidenced by the fact that highly degenerate gases expand negligibly in the direction of weakest optical confinement.

The imaging system. After magnetic focusing, the atoms are imaged with a 40 to $100 \mu$ s pulse of light resonant with the $F=2 \rightarrow F=3$ cycling transition. The magnification of our imaging system was calibrated using an optical micrometer target that was placed in a plane equivalent to the plane of the atoms with respect to the imaging system. This calibration was repeated several times with fractional uncertainty of $1 \%$ and is included in the systematic error of our thermometer.

The resolution of our imaging system, approximately $8 \mu \mathrm{m}$, corresponds to the Gaussian width of a thermal cloud at a temperature of $0.2 \mathrm{nK}$. We do not correct extracted temperatures for finite imaging resolution.
Details of the temperature fitting procedure. Atom column momentum densities $n_{p}$ are fitted to a Bose-enhanced momentum distribution $n_{p}=b+A g_{j}\left(e^{x}\right)$, with $g_{j}$ the Bose function (polylogarithm) of order $j$ and the argument

$$
x=\alpha-\left(p_{w}-p_{w 0}\right)^{2} / 2 k_{\mathrm{B}} T_{w}-\left(p_{t}-p_{t 0}\right)^{2} / 2 k_{\mathrm{B}} T_{t}
$$

For non-degenerate gases, $\alpha<0$ is a free parameter of the fit, along with the zero of momentum $\left(p_{w 0}, p_{t 0}\right)$, the background level $b$, peak level $A$, and temperatures $T_{w}$ and $T_{t}$ along the weak and tight in-plane axes of the optical trap, respectively. For degenerate gases, $\alpha=0$.

Generally, $T_{w}=T_{t}$ within the error of the fit; however, at low temperatures, several effects cause $T_{t}$ be an unreliable estimate of the gas temperature. Although the condensate expands primarily along the unfocused vertical direction, at the very low temperatures reported in this work, condensate expansion along the more tightly confined in-plane direction can be manifest. Also, the tilt of the trap in the tight direction is more difficult to calibrate, leading to a (fictitious) systematic upward shift in the apparent $T_{t}$ as explained above. Finally, at the lowest temperatures reported in this work, the semiclassical condition $k_{\mathrm{B}} T \gg \hbar \omega_{t}$ does not hold along the tight axis with trap frequency $\omega_{t}$. In contrast, the corresponding relation with $\omega_{w}$, the weak axis trap frequency, does hold $\left(\hbar\left\{\omega_{w}, \omega_{t}\right\} / k_{\mathrm{B}} \sim\{0.05,1\} \mathrm{nK}\right)$. Thus, in this work, we estimate temperatures by $T=T_{w}$ alone.

All fits exclude regions of the column density that include condensed atoms, and each fit was performed many times, varying both the size of the excluded central region and the order $j \in[0.5,2]$ of the Bose function to look for systematic shifts in the temperature. The high-momentum tails of the momentum distribution $n_{p}$ are insensitive to the particular Bose function employed $\left(g_{j}\left(e^{x}\right) \rightarrow e^{x}\right.$ as $\left.e^{x} \rightarrow 0\right)$. We used only fits where the variation of the temperature with respect to the size of the exclusion region and the order of the Bose function was negligible compared to other sources of systematic error.

The uncertainty with which individual fits estimate the temperature is consistent with fundamental sources of noise, photon and atom shot noise, and the short-time (same day) shot-to-shot variation of estimated temperatures was consistent with the single-shot noise-limited error estimated by the fitting routine. This justifies the use of an uncertainty-weighted average of many independent measurements to compute the precise low temperatures reported in this work.

Calculation of the entropy per particle. Calculations of the entropy per particle cannot rely on the local density approximation because the thermal phonon wavelength is far larger than the transverse extent of the condensed gas. We can, however, apply a one-dimensional local density approximation along the long axis of the condensate.

To calculate the entropy, we numerically solve for the Gross-Pitaevskii ground state $\Psi$ and Bogoliubov spectrum $\left\{\epsilon_{i}\right\}$ of small-amplitude excitations in a weakly interacting ${ }^{87} \mathrm{Rb}$ Bose gas with chemical potential $\mu$ in a trap with confinement in two dimensions, including the effects of gravity and anharmonicity, and no confinement in the third dimension. To do this calculation, we discretize the two-dimensional Gross-Pitaevskii equation and Bogoliubov equations on a Lagrange mesh based on the Hermite polynomials ${ }^{26,27}$. The Bogoliubov equations include the substitution $\mu \rightarrow \mu-\hbar^{2} k^{2} / 2 m$, for relevant values of the wavevector $k$, to account for excitations in the unconfined longitudinal direction. We verify that our calculation of the excitations $\left\{\epsilon_{i}\right\}$ are accurate by comparing to analytic results in regimes where they are available-for example, in the harmonic non-interacting limit and in the Thomas-Fermi limit with cylindrical symmetry.

The entropy and number of atoms per unit length can then be calculated using the equilibrium relations

$$
\begin{gathered}
\frac{S}{L}=\sum_{i} \frac{\left(\epsilon_{i}-\mu\right) / k_{\mathrm{B}} T}{\mathrm{e}^{\left(\epsilon_{i}-\mu\right) / k_{\mathrm{B}} T}-1}-\log \left(1-\mathrm{e}^{\left(\mu-\epsilon_{i}\right) / k_{\mathrm{B}} T}\right) \\
\frac{N}{L}=N_{\mathrm{c}}+\sum_{i} \frac{1}{\mathrm{e}^{\left(\epsilon_{i}-\mu\right) / k_{\mathrm{B}} T}-1}
\end{gathered}
$$

with $N_{c}$ the number of condensed atoms derived by integrating the density $|\Psi|^{2}$. By fixing $T$ and varying $\mu$ ( $\Psi$ and $\left\{\epsilon_{i}\right\}$ are recalculated for each value of $\mu$ ), we can consider the entropy and number per unit length as functions of $\mu: S(\mu) / L(\mu)$ and $N(\mu) / L(\mu)$

We use the local density approximation and Thomas-Fermi approximation along the long axis of our trap (with trap frequency $\omega_{z}$ ) to write the total entropy of the gas as

$$
S=\int_{-\infty}^{\infty} \frac{S}{L}\left(\mu_{\max }-\frac{1}{2} \omega_{z}^{2} z^{2}\right) \mathrm{d} z
$$

and the total number of atoms as

$$
N=\int_{-\infty}^{\infty} \frac{N}{L}\left(\mu_{\max }-\frac{1}{2} \omega_{z}^{2} z^{2}\right) \mathrm{d} z
$$


where $\mu_{\max }$ is chosen to yield the experimentally measured value of $N$. The entropy per particle of the gas is then $S / N$.

At the lowest trap depths, the rate at which the magnons are lost by forced evaporation becomes comparable to the rate at which non-condensed magnons are generated through thermalization. We speculate that the number of thermal excitations in the majority gas under these conditions is similarly lower than at the observed temperature in equilibrium. As such, our estimate of $S / N=1 \times 10^{-3} k_{\mathrm{B}}$ may be an underestimate of the true steady-state entropy.

Calculating $T_{\mathrm{c}}$ and $\mu$. The estimates of $T_{\mathrm{c}}$ and $\mu$ reported use standard formulae for Bose gases in the non-interacting and Thomas-Fermi limits, respectively ${ }^{28}$. The effects of trap anharmonicity and finite interactions on both $\mu$ and $T_{\mathrm{c}}$ were considered and are of the order of the systematic uncertainty in our temperature measurements.

Theoretical limits for decoherence cooling. Considering an ideal Bose gas in a harmonic trap with geometric-mean frequency $\omega$, the following relations for the saturated thermal atom number $N_{\text {th }}$, energy $E$, and heat capacity $C$, at the temperature $T$, apply:

$$
\begin{gathered}
N_{\text {th }}=\zeta(3)\left(\frac{k_{\mathrm{B}} T}{\hbar \omega}\right)^{3} \\
E=3 \zeta(4) \frac{\left(k_{\mathrm{B}} T\right)^{4}}{(\hbar \omega)^{3}}=\frac{3 \zeta(4)}{\zeta(3)} k_{\mathrm{B}} T N_{\mathrm{th}}
\end{gathered}
$$

$$
C=\frac{\mathrm{d} E}{\mathrm{~d} T}=12 k_{\mathrm{B}} \frac{\zeta(4)}{\zeta(3)} N_{\mathrm{th}}
$$

with $\zeta$ the Riemann zeta function. Suppose we transfer $\mathrm{d} N>0$ atoms to the minority spin state (where $\mathrm{d} N$ is far below the critical number for magnon condensation), let them thermalize, and eject them. To lowest order, the majority gas loses energy $\mathrm{d} E=-3 k_{\mathrm{B}} T \mathrm{~d} N$, and thus the temperature changes by $\mathrm{d} T=\mathrm{d} E / C=$ $-3\left(k_{\mathrm{B}} T\right) / C \mathrm{~d} N$. The non-condensed fraction changes as, using equations (1)-(3),

$$
\begin{aligned}
\mathrm{d}\left(\frac{N_{\mathrm{th}}}{N}\right) & =\frac{1}{N}\left[\mathrm{~d} N_{\mathrm{th}}+\frac{N_{\mathrm{th}}}{N} \mathrm{~d} N\right] \\
& =\frac{\mathrm{d} N}{N}\left[-\frac{3 \zeta(3)}{4 \zeta(4)}+\frac{N_{\mathrm{th}}}{N}\right]
\end{aligned}
$$

Thus, we must have $N_{\mathrm{th}} / N<(3 \zeta(3)) /(4 \zeta(4))=0.83$ to reduce the non-condensed fraction through such cooling. This corresponds to, for the case under consideration, $T / T_{\mathrm{c}}<(0.83)^{1 / 3}=0.94$.

\section{References}

26. Baye, D. \& Heenen, P.-H. Generalised meshes for quantum mechanical problems. J. Phys. A. 19, 2041-2059 (1986).

27. McPeake, D. Superfluidity, Collective Excitations and Nonlinear Dynamics of Bose-Einstein Condensates Thesis, Queen's University, Belfast (2002).

28. Pitaevskii, L. P. \& Stringari, S. Bose-Einstein Condensation (Oxford Univ. Press, 2003). 


\section{CORRIGENDUM}

\section{Thermometry and cooling of a Bose gas to 0.02 times the condensation temperature}

Ryan Olf, Fang Fang, G. Edward Marti, Andrew MacRae and Dan M. Stamper-Kurn

Nature Physics http://dx.doi.org/10.1038/nphys3408 (2015); published online 20 July 2015; corrected online 20 August 2015.

In the version of this Letter originally published ref. 2 was mistakenly reproduced as ref. 26. Ref. 26 has been removed from all versions of the Letter. 